\title{
Improvements for electromechanical oscillation mode estimation via subspace identification methods
}

\author{
Sheng Ding ${ }^{1}$ and Hongliang Huang ${ }^{2^{*}}$
}

\begin{abstract}
Two improvements of power system electromechanical oscillation estimation via subspace identification are proposed in this paper. Singular value-based criteria are introduced not only to improve the computation efficiency for determining the model order of the estimated system but also to improve the accuracy of the estimates. A mode matching method based on the characteristics of accurate mode shape estimates is proposed to ensure the correctness of the estimates. Numerical results from three different scale power systems illustrate the feasibility of the proposed methods.
\end{abstract}

Keywords: Electromechanical oscillation; Subspace identification methods; Model order selection; Mode matching; Singular value criterion

\section{Introduction}

Electromechanical oscillations in power system are becoming a serious factor to limit power transfer in interconnected power systems in China. The Southern Grid as well as the North China Grid is often reported to be suffering from inter-area oscillations [1,2]. Many blackouts either directly or indirectly caused by low-frequency oscillation or occurrences of low-frequency oscillation have been reported worldwide [3]. Among them, blackouts that occurred across Western Canada, the Western United States, and Northwest Mexico on 10 August 1996 [4] and in the eastern United States and parts of Canada on 14 August 2003 [5] are well known. These two outages show the potential hazards that inter-area oscillation modes might bring to the whole power grid.

Many efforts have been focusing on the electromechanical oscillation analysis so far. Since analysis based on first-principle modeling is not accurate enough [4], identification methods are preferred to estimate the properties of the modes. Among them, subspace methods are becoming attractive not only because the state space form of the system is very convenient for estimation,

\footnotetext{
* Correspondence: davidhwang.zju@gmail.com

${ }^{2}$ College of Electrical Engineering, Zhejiang University, Hangzhou 310027, China Full list of author information is available at the end of the article
}

prediction, and control [6] but also because they have numerical simplicity and robustness and are suitable for multiple-input and multiple-output (MIMO) system, such as large power system. In [7], subspace methods are applied to estimate the electromechanical modes using ambient data. In [8], the Monte Carlo simulation results show that compared with Prony method and Yule-Walker method, canonical variate analysis (CVA) as well as numerical algorithms for Subspace State Space System Identification (N4SID), two subspace methods, are more accurate in estimating frequencies and damping ratios of low-frequency oscillation modes. In [9], N4SID is also more accurate using ring-down data. Subspace methods are also developed to estimate mode shapes. It is concluded that the mode shapes obtained by subspace methods are more accurate compared with the ones obtained by other methods such as transfer function (TF), spectral frequency domain decomposition (FDD) [10]. Furthermore, no extra algorithm is required to calculate the mode shapes if the subspace method is applied.

However, there may still have some important considerations neglected when subspace methods are applied to estimate the electromechanical oscillations. The two main problems will be investigated in this paper. One is how to determine the proper order of the estimated 
system matrix both efficiently and accurately. Overparameterization or under-parameterization could result in inaccurate estimates. As yet, two information criteria have been widely used to determine the system order: Akaike's information criterion (AIC) [11] and Bayesian information criterion (BIC) [12]. Both criteria can be used to solve model order selection problem for autoregressivemoving-average (ARMA) model or other identification methods successfully [13]. In [8] and many other references, AIC is also applied to determine the model order for subspace identification method. However, the model order should be determined after a sequence of tests for each order if AIC or BIC is applied [14]. An efficient computation way for model order selection for subspace methods can be based on the singular values of the Hankel matrix which are estimated in the algorithm of subspace identification methods. In this paper, singular value-based criteria are introduced to improve computation efficiency and the accuracy of the estimates.

The other is the mode matching problem. The frequency range of an electromechanical oscillation mode is about $0.2 \sim 2.5 \mathrm{~Hz}$. It can be noted that often there are several modes of interest whose frequencies are quite close. For example, in [15] the author shows that the oscillation modes existing in the WECC among the frequencies of two modes are 0.78 and $0.8 \mathrm{~Hz}$. Furthermore, the estimated mode will be interfered with other frequency-neighboring modes. In this content, the correctness of the estimated mode should be ensured. In this paper, a method of mode matching is proposed for subspace identification methods. By extracting the characteristic information from the estimated mode shape, the estimated mode can be determined to which mode it corresponds. It is also shown that the aggregation method can help simplify the extraction.

The paper is organized as follows: A general procedure of estimation based on subspace identification methods are described systematically in Section 2. Model order selection is discussed in Section 3. A method of mode matching via mode shapes is shown in Section 4. The simulation results are shown in Section 5. Conclusions are drawn in Section 6.

\section{Mode estimation via subspace identification methods}

Many identification methods as well as signal processing techniques are used to estimate the electromechanical oscillation modes based on the measurements [16]. Among them, subspace identification methods are more popular due to the following reasons:

1. State space form of the identified system is very convenient for estimation, prediction, and control [6]. Also, subspace identification methods have numerical simplicity and robustness and are suitable for MIMO system, such as large power system. Several oscillation modes of interests can be estimated accurately.

2. Subspace methods are able to obtain accurate modal properties (frequency and damping ratio) using ambient and ring-down data [9]. Ambient data correspond to stationary normal operating condition with small variations due to random load variations in the whole system, while ring-down data are observed when some radical changes occur in the system, e.g., a transmission line tripping or a generator tripping [17]. So subspace identification methods have a better tracking ability for a practical power grid monitoring with both of the two types of data generated by a real power system.

3. Subspace methods can also give accurate estimates of mode shapes giving more information about the oscillation modes like the dominant oscillation paths and the source. This is due to the fact that there is no approximation when mode shapes are calculated [10].

According to the above advantages, subspace identification methods are becoming more attractive in electromechanical oscillation mode estimation. Usually, according to [8] and [18], the procedure of electromechanical modes estimation via subspace identification methods includes phase data collection, signal pre-processing, modal properties via subspace identification methods, and accuracy evaluation (shown in Figure 1). All these steps will be introduced in the following sections. Two improvements are also shown in red dashed line block in Figure 1. Singular value criterion (SVC), instead of information criteria like AIC and BIC, will efficiently give a more proper model order to make the estimates more accurate. Mode matching can help match the estimated modes with the ones of interest. Both the improvements will be introduced detailedly in Section 3 and Section 4.

\subsection{Phase data collection}

Measurements from wide area are collected for electromechanical modes estimation. Wide area measurement systems (WAMS) are used to bring synchrophasor measurement-based information into the control center from different parts of inter-connected power systems [19]. Ambient or ring-down measurements from phasor measurement units (PMUs) are sent to phasor data concentrator (PDC) located in control center through digital communication channels [17]. Thus, one can select measurements from several channels where the modes of interest can be easily observed. 


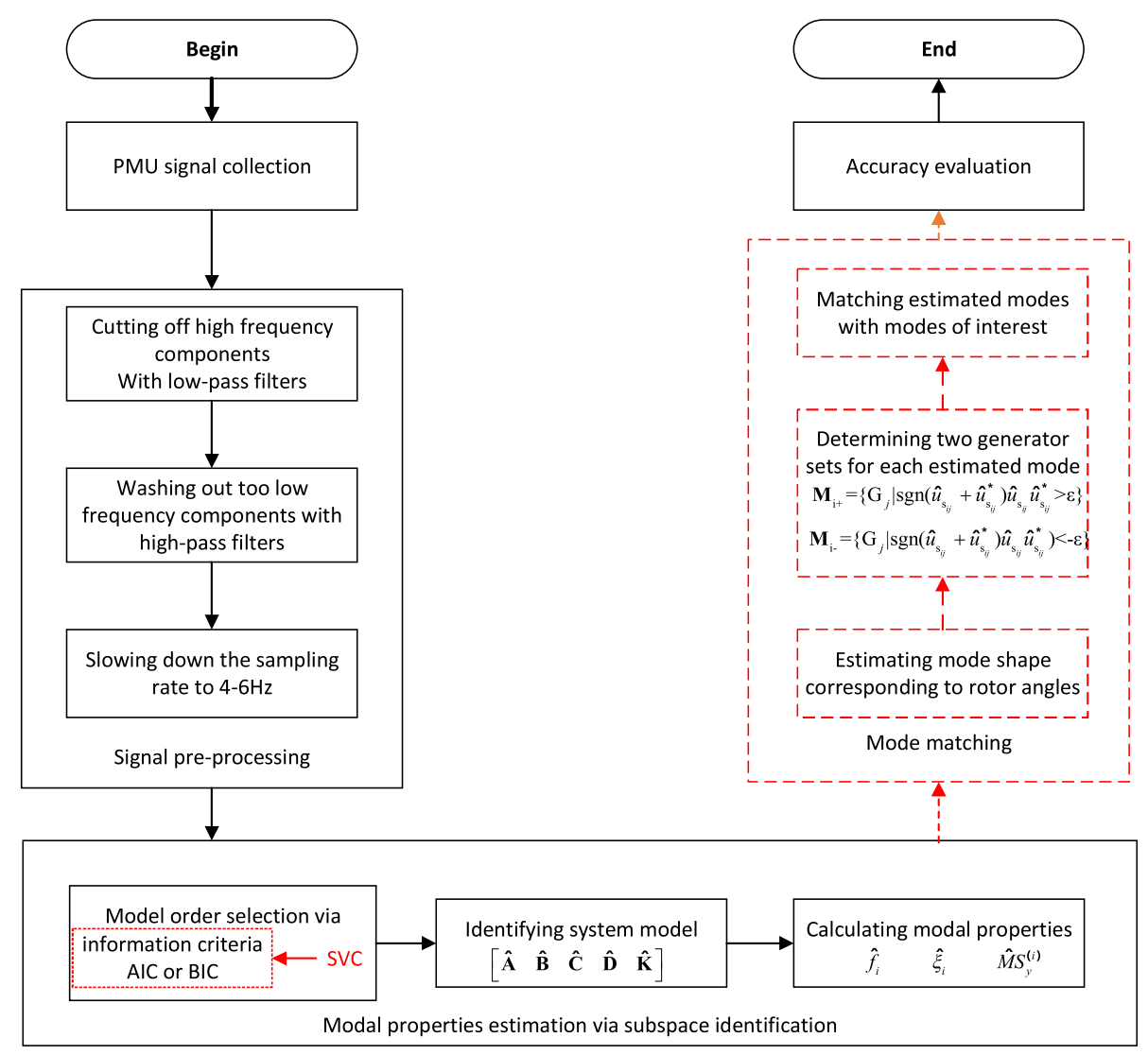

Figure 1 Procedure of electromechanical oscillation mode estimation via subspace methods.

\subsection{Signal pre-processing}

The aim of pre-processing PMU data is to extract the features of electromechanical modes whose frequency covers the range from 0.2 to $2.5 \mathrm{~Hz}$. Thus, more accurate estimates can be obtained. Several steps should be taken into consideration [8]. First, several low-pass filters can cut off high-frequency components. Second, highpass filters should be used to wash out components with too low frequencies. Last but not the least, decimation should be conducted to slow down the sampling rate (20 $30 \mathrm{samples/s)}$ to the optimum. This is due to the fact that high sampling rate will make the discrete poles sensitive to noise and the poles closer to each other according to [20]. Too low sampling rate will, however, make the signal distorted according the Shannon Sampling Theorem. An optimal sampling rate is usually about $4 \sim 6 \mathrm{~Hz}$ for electromechanical modes estimation.

\subsection{Modal properties estimation via subspace identification}

The aim of subspace identification is to identify a reduced-order linear model for power system. Electromechanical oscillation modes can thus be estimated from this model. In this paper, since only ambient data are used, i.e., the system is perturbed by small load variations, the identified linearized power system via subspace identification methods can be written as [10] follows:

$$
\left\{\begin{array}{c}
\dot{\hat{\hat{x}}}(k+1)=\hat{\hat{\mathbf{A}}} \hat{\hat{x}}(k)+\mathbf{K} \underline{e}(k) \\
\underline{y}(k)=\hat{\mathbf{C}} \underline{\hat{x}}(k)+\underline{e}(k)
\end{array}\right.
$$

where $\underline{\hat{x}}$ is the state vector. $y$ is the output vector. $\underline{e}$ is the input noise. $\widehat{\mathbf{A}}, \widehat{\mathbf{C}}$, and $\overline{\mathbf{K}}$ are the estimated system matrix, the output matrix, and the input noise gain matrix, respectively.

If $\hat{Z}_{\mathrm{i}}$ is the estimate of the discrete eigenvalue, one can obtain the corresponding continuous eigenvalue:

$$
\hat{\lambda}_{i}=f_{s} \ln \left(\hat{z}_{i}\right)
$$

where $f_{s}$ is the sampling rate. The corresponding estimated frequency and damping ratio can be calculated by the following:

$$
\hat{f}_{i}=\frac{\operatorname{Im}\left(\hat{\lambda}_{i}\right)}{2 \pi}
$$




$$
\hat{\xi}_{i}=-\frac{\operatorname{Re}\left(\hat{\lambda}_{i}\right)}{\left|\hat{\lambda}_{i}\right|}
$$

Once the corresponding right eigenvector $\hat{\underline{u}}_{i}$ is found, the relative mode shape of measured output can be defined as [21] follows:

$$
\widehat{\mathbf{M}} \mathbf{S}_{y}^{(i)}=\hat{\mathbf{C}} \underline{\hat{u}}_{i}
$$

\subsection{Accuracy evaluation}

Load variations can be seen following Gaussian distribution [22]. Based on this assumption, the accuracy of the estimates has been evaluated. Bootstrap method has been applied to determine the uncertainties in subspace identification methods by resampling residuals [23]. For electromechanical oscillation estimation, since the length of measured data is limited, some Monte Carlo methods like bootstrap are preferred to evaluate the estimates [24-26].

In this paper, the model order selection will be investigated. An efficient method, SVC, instead of AIC and $\mathrm{BIC}$, gives more accurate estimates. A step of mode matching is also added into the procedure to find out the corresponding mode from all the estimated modes (shown in Figure 1).

\section{Model order selection for subspace identification method}

Model order has great influence on the accuracy of estimated modal properties. Higher order or lower order usually results in over-parameterization or under-parameterization. Both situations will make the estimates inaccurate. Usually, AIC [11,27] and BIC [12] are used to determine the model order for ARMA or ARMAX model. For subspace identification methods, however, AIC or BIC might not work quite efficiently. An efficient computation way to determine the model order is based on the singular values of the weighted Hankel matrix. Two singular value-based methods for model order selection are thus adopted for subspace identification methods to improve the computation efficiency in this section. SVC is suggested for subspace identification methods.

\subsection{Information criteria}

Information criteria (IC) are referred to as penalized loglikelihood criteria where the penalty term depends on the number of free parameters in the model and the number of observations [13]. They can be written in a general form:

$$
\mathrm{IC}(n)=-2 \sum_{i=1}^{\mathrm{N}} \log f\left(\mathbf{x}_{\mathrm{i}} \mid \hat{\boldsymbol{\theta}}_{n}\right)+\mathrm{d}(n) \mathrm{C}(\mathrm{N})
$$

where $f\left(\mathrm{x}_{\mathrm{i}} \underline{\hat{\boldsymbol{\theta}}}_{n}\right), i=1, \ldots, \mathrm{N}$ describes the conditional probability density of the observations $x_{1}, x_{2}, \ldots, x_{N}$; $\mathrm{C}(\mathrm{N})$ is an increasing function of the observations number $\mathrm{N}$, and $\underline{\hat{\boldsymbol{\theta}}}_{n}$ is the estimator for the unknown model parameter based on the observations. $\mathrm{d}(n)$ is the number of the parameters in $\hat{\boldsymbol{\theta}}_{n}$. The optimal order choice is such that $\hat{n}_{\mathrm{IC}}=\operatorname{argmin} I \mathrm{C}(n)$. The widely used information criteria, AIC [27] and BIC [12], are defined as follows:

$$
\begin{aligned}
& \operatorname{AIC}(n)=-2 \sum_{\mathrm{i}=1}^{\mathrm{N}} \log \mathrm{f}\left(x_{\mathrm{i}} \mid \underline{\boldsymbol{\theta}}_{n}\right)+2 \mathrm{~d}(n) \\
& \operatorname{BIC}(n)=-2 \sum_{\mathrm{i}=1}^{\mathrm{N}} \log f\left(x_{\mathrm{i}} \mid \underline{\hat{\boldsymbol{\theta}}}_{n}\right)+\mathrm{d}(n) \times \log \mathrm{N}
\end{aligned}
$$

It is stated in [13] that BIC can be used in ARMA model. In [8] and many other references, AIC is applied to choose the order for the system model identified by subspace methods. Noting that from (7) and (8), one can see that before calculating $\operatorname{AIC}(n)$ and $\operatorname{BIC}(n), \underline{\hat{\boldsymbol{\theta}}}_{n}$ should be obtained.

\subsection{Singular value-based criteria}

Recall the state space model (1), for open loop system, the observability matrix can be written as follows:

$$
\mathbf{r}_{\mathrm{f}}=\left[\begin{array}{llll}
\mathbf{C}^{\top} & \mathbf{A}^{\top} \mathbf{C}^{\top} & \cdots & \left(\mathbf{A}^{\mathrm{f}-1}\right)^{\top} \mathbf{C}^{\top}
\end{array}\right]^{\top}
$$

Also, the controllability matrix can be written as follows:

$$
\mathbf{L}_{\rho}=\left[\begin{array}{llll}
\mathbf{B} & \mathbf{A B} & \cdots & \mathbf{A}^{\rho-1} \mathbf{B}
\end{array}\right]
$$

The Hankel matrix $\mathrm{H}_{f p}$ is the product of the observability matrix and controllability matrix:

$$
\mathbf{H}_{f p} \triangleq \Gamma_{f} \mathbf{L}_{p}=\left[\begin{array}{cccc}
\mathbf{C B} & \mathbf{C A B} & \cdots & \mathbf{C A}^{p-1} \mathbf{B} \\
\mathbf{C A B} & \mathbf{C A}^{2} \mathbf{B} & \cdots & \mathbf{C A}^{p} \mathbf{B} \\
\vdots & \vdots & \cdots & \vdots \\
\mathbf{C A}^{f-1} \mathbf{B} & \mathbf{C A}^{f} \mathbf{B} & \cdots & \mathbf{C A}^{f+p-2} \mathbf{B}
\end{array}\right]
$$

For subspace identification methods, the estimated Hankel matrix $\widehat{\mathbf{H}}_{f p}$ can be obtained by the measurement data. Usually, $p$ is a large positive integer due to the high-order ARX (HOARX) model used in the asymptotic methods [6]. Therefore, singular value decomposition is performed on the weighted Hankel matrix and 
then a new matrix with lower rank $\widehat{n}$ can be approximately obtained:

$$
\mathbf{W}_{f} \widehat{\mathbf{H}}_{f p} \mathbf{W}_{p}=\widehat{\mathbf{U}} \widehat{\Sigma} \widehat{\mathbf{V}}^{\top} \simeq \widehat{\mathbf{U}}_{\hat{n}} \widehat{\Sigma}_{\hat{n}} \widehat{\mathbf{V}}_{\hat{n}}^{T}
$$

where $\mathbf{W}_{f}$ and $\mathbf{W}_{p}$ are the weight matrices. They vary with different subspace identification methods. One can find $\mathbf{W}_{f}$ and $\mathbf{W}_{p}$ for CCA, MOESP, and N4SID in [14].

Suppose there are $m$ outputs and no input in the system. There are $\min (f m, p m)$ singular values in $\widehat{\Sigma}$, and $\widehat{\sigma}_{\mathrm{i}}$ is the $i$ th largest singular value in $\hat{\Sigma}$. The difference between $\widehat{\mathbf{U}} \widehat{\Sigma} \widehat{\mathbf{V}}^{T}$ and $\widehat{\mathbf{U}}_{\hat{n}} \widehat{\Sigma}_{\hat{n}} \widehat{\mathbf{V}}_{\hat{n}}^{T}$ can be defined in both Frobenius norm and Eulclidean norm:

$$
\begin{aligned}
& \hat{\mathbf{U}} \hat{\mathbf{S}} \hat{\mathbf{V}}^{\top}-\hat{\mathbf{U}}_{n} \hat{\mathbf{S}}_{n} \hat{\mathbf{V}}_{n}^{T} \|_{\mathrm{F}}=\sqrt{\sum_{j=n+1}^{\min (f s, p s)} \hat{\sigma}_{j}^{2}} \\
& \left\|\hat{\mathbf{U}} \hat{\mathbf{S}} \hat{\mathbf{V}}^{T}-\hat{\mathbf{U}}_{n} \hat{\mathbf{S}}_{n} \hat{\mathbf{V}}_{n}^{T}\right\|_{2}=\hat{\sigma}_{n+1}
\end{aligned}
$$

Similar to AIC and BIC, combining the penalty term which punishes the high model orders, novel information criterion (NIC) and SVC can be defined as [28] follows:

$$
\begin{aligned}
& \operatorname{NIC}(n)=\sum_{j=n+1}^{\min (f s, p s)} \widehat{\sigma}_{j}^{2}+d(n) \mathrm{C}(\mathrm{N}) / \mathrm{N} \\
& \operatorname{SVC}(n)=\widehat{\sigma}^{2}{ }_{n+1}+d(n) \mathrm{C}(\mathrm{N}) / \mathrm{N}
\end{aligned}
$$

where $d(n) \mathrm{C}(\mathrm{N}) / \mathrm{N}$ is the penalty term. Compared with $\mathrm{AIC}$ and BIC, the computation efficiency of NIC and SVC is highly improved due to the fact that the singular values are estimated in the algorithm in subspace methods. Note that NIC and SVC use Frobenius and Eulclidean norm to measure the difference between $\widehat{\mathbf{U}} \widehat{\Sigma} \widehat{\mathbf{V}}^{T}$ and $\widehat{\mathbf{U}}_{\hat{n}} \widehat{\Sigma}_{\hat{n}} \widehat{\mathbf{V}}_{\hat{n}}^{T}$. In comparison with NIC, SVC is less influenced by the row and column indices of the Hankel matrix $\mathrm{f}$ and $p$ since $\operatorname{SVC}(n)$ takes the largest neglected singular value $\hat{\sigma}_{n+1}$ into consideration while $\mathrm{NIC}(n)$ takes all the neglected singular values [28]. In this paper, SVC is preferred in the following discussion.

Recall that $\mathrm{f}$ and $p$ are the row and column indices of the Hankel matrix respectively. The accuracy of the estimation of the model order $\widehat{n}$ heavily depends on the values of $\mathrm{f}$ and $p$. It is required that index $\mathrm{p}$ tend to infinity for consistency of the estimates [14]. Fortunately, $p$ can reduce to $\widehat{p}_{\text {AIC }}$, the estimated order of autoregressive (AR) model according to AIC, which is the well-known high-order AR model in the asymptotic methods [29]. Usually, we have the following:

$$
f=p=\widehat{p}_{\text {AIC }}
$$

Mathematically, the optimal model order can be described as follows:

$$
\widehat{\mathrm{n}}_{\mathrm{SVC}}=\underset{0 \leq n \leq \min (f m, p m)}{\arg \min } \operatorname{SVC}(n)
$$

It is proved in [14] that if $\operatorname{limC}(\mathrm{N}) / \mathrm{N} \rightarrow 0$ and $\operatorname{liminfC}(\mathrm{N}) /($ fploglog $N) \rightarrow \infty$, then $\widehat{\mathrm{n}}_{\mathrm{SVC}} \rightarrow \mathrm{n}_{0}$, where $\mathrm{n}_{0}$ is the order of the reduced system. An obvious advantage of SVC over information criteria is that the system order can be determined by calculating the singular values of $\mathbf{W}_{f} \widehat{\mathbf{H}}_{f p} \mathbf{W}_{p}$ which is already conducted in subspace identification methods. Many details about SVC can be found in [14].

So far, three information criteria are introduced in this section. By SVC, the computation efficiency of model order selection has been highly improved. The accuracies of the three criteria will be examined with simulation examples and one may find that SVC also improves the accuracy of the estimates.

\section{Mode matching}

There are many electromechanical oscillation modes with similar frequency in inter-connected power systems. Practically, only several lightly damped modes which can be found by first-principle modeling are of interest. However, many estimated modes are quite closer so that one cannot determine the mode corresponding to the one of interest. The importance of mode matching is to ensure the correctness of the estimates, i.e., the estimated mode should correspond to the mode of interest.

The mode matching method proposed for subspace identification method in this paper is based on the estimated mode shape, i.e., the relative magnitude and phasing, of the oscillation throughout the system [30]. The right eigenvector gives the 'mode shape', i.e., the relative activity of the state variables when a particular mode is excited [31]. The amplitude of the mode shape indicates how much the generators participate in the mode. The angle of the mode shape indicates the two swing-against groups. In this paper, two sets containing mainly swinging against generator groups can be determined by the estimated mode shape. Furthermore, inertia aggregation method is accurate enough to aggregate the coherent generators in the system for calculation simplicity.

\subsection{Modal analysis}

Suppose there are $\mathrm{m}$ generators and $\mathrm{n}_{\mathrm{b}}$ buses in the system. Voltage angles of $\mathrm{m}$ buses are measured as the 
system outputs. The electromechanical model can be linearized at the operating point and written as a state space form [30]:

$$
\left\{\begin{array}{l}
\underline{\dot{x}}=\mathbf{A} \underline{x} \\
\underline{y}=\mathbf{C} \underline{x}
\end{array}\right.
$$

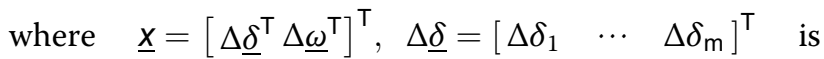
the rotor angle deviation vector in electrical radians, $\Delta \underline{\omega}=\left[\begin{array}{lll}\Delta \omega_{1} & \cdots & \Delta \omega_{\mathrm{m}}\end{array}\right]^{\top}$ is the per unit speed deviation vector, and $\underline{y}$ is the bus voltage phase angle vector.

The system matrix $\mathbf{A}$ is as follows:

$$
\mathbf{A}=\left[\begin{array}{cc}
\mathbf{0} & \omega_{0} \mathbf{I} \\
-\mathbf{M}^{-1} \mathbf{K}_{s} & -\mathbf{M}^{-1} \mathbf{K}_{D}
\end{array}\right]
$$

where $\mathbf{M}=\operatorname{diag}\left(2 \mathrm{H}_{1}, \cdots, 2 \mathrm{H}_{m}\right)$ is the matrix of aggregated generators' inertias. $\mathbf{K}_{S}$ and $\mathbf{K}_{D}$ are called synchronizing and damping torque coefficient matrices, respectively. In Appendix 1, it is shown how to find the entry of $\mathbf{K}_{S}$.

For simplicity, damping terms are neglected $\left(\mathbf{K}_{D} \approx \mathbf{0}\right)$. Rewrite the system matrix:

$$
\tilde{\mathbf{A}}=\left[\begin{array}{cc}
\mathbf{0} & \omega_{0} \mathbf{I} \\
-\mathbf{M}^{-1} \mathbf{K}_{s} & \mathbf{0}
\end{array}\right]
$$

By definition:

$$
\tilde{\mathbf{A}} \underline{u}_{i}=\lambda_{i} \underline{u}_{i} \Rightarrow\left[\begin{array}{cc}
\mathbf{0} & \omega_{0} \mathbf{l} \\
-\mathbf{M}^{-1} \mathbf{K}_{s} & \mathbf{0}
\end{array}\right]\left[\begin{array}{l}
\underline{u}_{i 1} \\
\underline{u}_{i 2}
\end{array}\right]=\lambda_{i}\left[\begin{array}{l}
\underline{u}_{i 1} \\
\underline{u}_{i 2}
\end{array}\right]
$$

where $\lambda_{i}$ is the ith eigenvalue of $\mathbf{A} ; \underline{u}_{i}$ is the corresponding eigenvector. Rearranging (4-4), one can obtain:

$$
\left\{\begin{array}{c}
\omega_{0} \mathbf{M}^{-1} \mathbf{K}_{s} \underline{u}_{i 2}=-\lambda_{i}^{2} \underline{u}_{i 2} \\
\underline{u}_{i 1}=\frac{\omega_{0} \mathbf{I}}{\lambda_{i}} \underline{u}_{i 2}
\end{array}\right.
$$

Thus, $-\lambda_{i}^{2}$ and $\underline{u}_{i 2}$ are eigenvalue and eigenvector of $\omega_{0} \mathbf{M}^{-1} \mathbf{K}_{s}$, respectively. From (23), $\mathrm{u}_{\mathrm{i} 1}$, mode shapes of low-frequency oscillation corresponding to generators' power angles can thus be found. Note that the main oscillatory characteristics for the mode shapes from (21) are maintained for the mode shapes of (20) [21]. All the elements in $u_{i 1}$ are real, while the elements would be complex if damping terms are not neglected. To this extent, (21) will give an easy way to find the oscillatory characteristics.

In most cases, voltage phase angles of the buses are taken as output signals for electromechanical oscillation

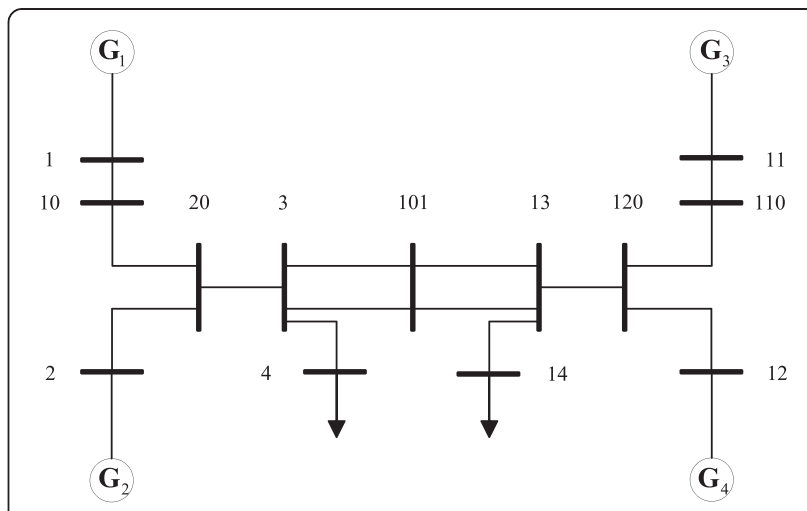

Figure 2 Schematic diagram of Four-Machine System.

estimation. Suppose the outputs of the system are voltage phase angles, one will have the following:

$$
\mathbf{C}=\left[\begin{array}{ll}
\mathbf{C}_{\theta \delta} & \mathbf{0}
\end{array}\right]
$$

where

$$
\mathbf{C}_{\theta \delta}=\left[\begin{array}{ccc}
\frac{\partial \theta_{1}}{\partial \delta_{1}} & \cdots & \frac{\partial \theta_{1}}{\partial \delta_{\mathrm{m}}} \\
\vdots & \ddots & \vdots \\
\frac{\partial \dot{\theta}_{\mathrm{m}}}{\partial \delta_{1}} & \cdots & \frac{\partial \dot{\theta}_{\mathrm{m}}}{\partial \delta_{\mathrm{m}}}
\end{array}\right]
$$

In Appendix 2, it is shown how to find entry of $\mathbf{C}_{\theta \delta}$ in detail.

\subsection{Mode matching method}

Obviously, the estimated mode shape $\widehat{\underline{u}}_{i 1}$ can be determined as follows:

$$
\widehat{\underline{u}}_{i 1}=\widehat{\mathrm{M}} \mathrm{S}_{\delta}^{(i)}=\mathbf{C}^{-1}{ }_{\theta \delta} \widehat{\mathrm{M}} \mathbf{S}_{y}^{(i)}
$$

$\widehat{\underline{u}}_{i 1}$ can be scaled by dividing the largest amplitude of its entries. Note the normalized vector as $\widehat{\underline{u}}_{s_{i}}$ and the jth entry in $\widehat{\underline{u}}_{\mathrm{s}_{i}}$ as $\widehat{u}_{\mathrm{s}_{i j}}, j=1, \ldots, \mathrm{m}$. The amplitude of $\widehat{u}_{\mathrm{s}_{i j}}$

Table 1 Estimated modes corresponding to different criteria

\begin{tabular}{lllllll}
\hline IC & Mode & Freq (Hz) & DR (\%) & $\mathbf{M}_{+}$ & $\mathbf{M}_{-}$ & Match result \\
\hline AIC order: 13 & EST 1 & 0.962 & 18.84 & $\mathrm{G}_{1}$ & $\mathrm{G}_{2}$ & Local mode 1 \\
& EST 2 & 0.964 & 17.89 & $\mathrm{G}_{4}$ & $\mathrm{G}_{3}$ & Local mode 2 \\
& EST 3 & 0.589 & 11.61 & $\mathrm{G}_{1} \mathrm{G}_{2}$ & $\mathrm{G}_{3} \mathrm{G}_{4}$ & Inter-area \\
BIC order: 13 & EST 1 & 0.964 & 17.89 & $\mathrm{G}_{4}$ & $\mathrm{G}_{3}$ & Local mode 2 \\
& EST 2 & 0.962 & 18.84 & $\mathrm{G}_{1}$ & $\mathrm{G}_{2}$ & Local mode 1 \\
& EST 3 & 0.589 & 11.61 & $\mathrm{G}_{1} \mathrm{G}_{2}$ & $\mathrm{G}_{3} \mathrm{G}_{4}$ & Inter-area \\
SVC order: 12 & EST 1 & 0.992 & 18.95 & $\mathrm{G}_{1}$ & $\mathrm{G}_{2}$ & Local mode 1 \\
& EST 2 & 0.999 & 18.12 & $\mathrm{G}_{4}$ & $\mathrm{G}_{3}$ & Local mode 2 \\
& EST 3 & 0.567 & 11.23 & $\mathrm{G}_{3}$ & $\mathrm{G}_{1} \mathrm{G}_{2}$ & Inter-area mode \\
\hline
\end{tabular}




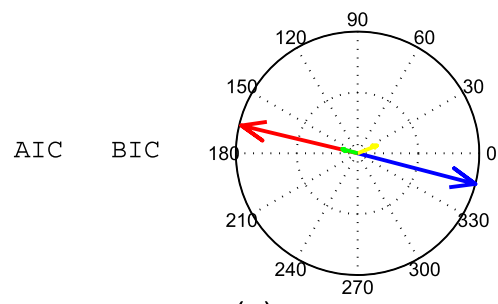

(a) estimated Local Mode 1

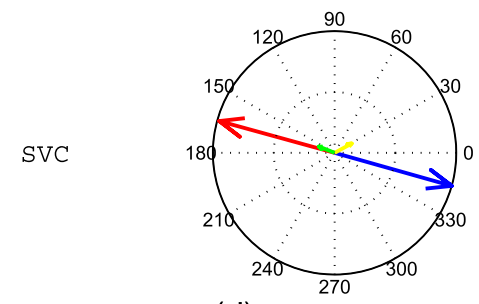

(d) estimated Local Mode 1

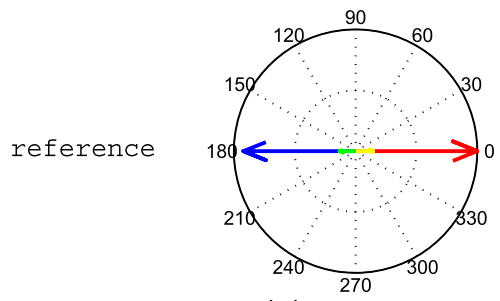

(g) Local Mode 1

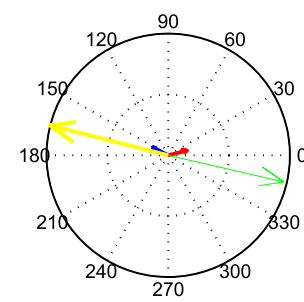

(b) estimated Local Mode 2

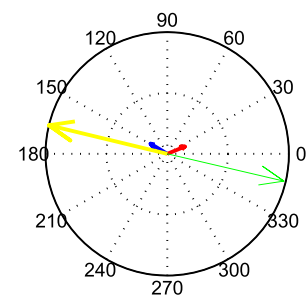

(e) estimated Local Mode 2

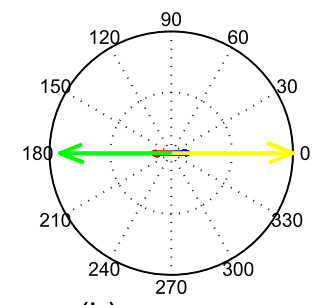

(h) Local Mode 2

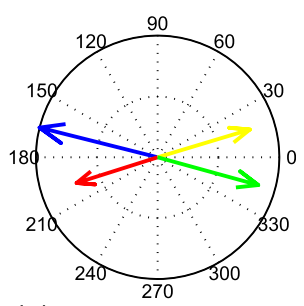

(c) estimated Inter Area Mode

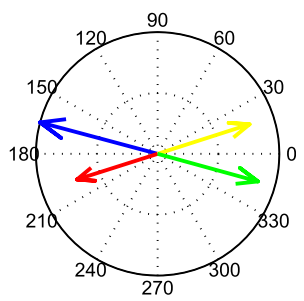

(f) estimated Inter Area Mode

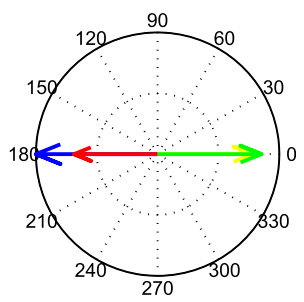

(i) Inter Area Mode

$$
-\mathrm{G} 1-\mathrm{G} 2-\mathrm{G} 3-\mathrm{G} 4
$$

Figure 3 Estimated and reference mode shapes of oscillation modes. (a) Estimated local model 1 via AIC or BIC. (b) Estimated local mode 2 via AIC or BIC. (c) Estimated inter-area mode via AIC or BIC. (d) Estimated local mode 1 via SVC. (e) Estimated local mode 2 via SVC. (f) Estimated inter-area mode via SVC. (g) Local mode 1. (h) Local mode 2. (i) Inter-area mode.

provides the information on the magnitude of the $i$ th mode in the state $\Delta \delta_{j}[16]$ i.e., the amplitude of $\widehat{u}_{s_{i j}}$ indicates how much the jth generator participating the ith mode. The angle of $\widehat{u}_{s_{i j}}$ provides the information on the phase of the $i$ th mode in the state $\Delta \delta_{j}$ [16] i.e., the angle of $\widehat{u}_{s_{i j}}$ indicates the pattern of generator swings for the ith mode. In this paper, the sign of the real part of $\widehat{u}_{\mathrm{s} i j}$ can help divide generators into one of the two swinging-against groups. Thus, two sets $\mathbf{M}_{i_{+}}$and $\mathbf{M}_{\mathbf{i}_{-}}$ containing mainly swinging against generators can be determined by $\widehat{u}_{s_{i j}}$

$$
\begin{aligned}
& \mathbf{M}_{\mathrm{i}+}=\left\{\mathrm{G}_{j} \mid \operatorname{sgn}\left(\widehat{u}_{\mathrm{s}_{i j}}+\widehat{u}_{\mathrm{s}_{i j}}^{*}\right) \widehat{u}_{\mathrm{s}_{i j}} \widehat{u}_{\mathrm{s}_{i j}}^{*}>\varepsilon\right\} \\
& \left.\mathbf{M}_{\mathrm{i}-}=\left\{\mathrm{G}_{j} \mid \operatorname{sgn}\left(\widehat{u}_{\mathrm{s}_{i j}}+\widehat{u}_{\mathrm{s}_{i j}}^{*}\right) \widehat{u}_{\mathrm{s}_{\mathrm{i} j}} \widehat{u}_{\mathrm{s}_{i j}}^{*}\right)<-\varepsilon\right\}
\end{aligned}
$$

\begin{tabular}{|c|c|c|c|c|c|c|}
\hline \multirow[t]{2}{*}{ Mode } & & \multirow[t]{2}{*}{ Reference value } & \multicolumn{2}{|l|}{ AIC BIC } & \multicolumn{2}{|l|}{ SVC } \\
\hline & & & Uncertainty & RMSE & Uncertainty & RMSE \\
\hline \multirow[t]{2}{*}{ Local mode 1} & Freq $(\mathrm{Hz})$ & 1.056 & $0.962 \pm 0.021$ & 0.094 & $0.992 \pm 0.020$ & 0.065 \\
\hline & DR (\%) & 19.87 & $18.86 \pm 3.88$ & 2.18 & $18.87 \pm 3.68$ & 2.13 \\
\hline \multirow[t]{2}{*}{ Local mode 2} & Freq $(\mathrm{Hz})$ & 1.071 & $0.964 \pm 0.021$ & 0.108 & $0.999 \pm 0.020$ & 0.073 \\
\hline & DR (\%) & 19.57 & $18.13 \pm 4.16$ & 2.41 & $18.39 \pm 4.12$ & 2.26 \\
\hline \multirow[t]{2}{*}{ Inter-area } & Freq $(\mathrm{Hz})$ & 0.565 & $0.589 \pm 0.018$ & 0.025 & $0.567 \pm 0.018$ & 0.009 \\
\hline & DR (\%) & 10.28 & $11.51 \pm 3.04$ & 2.16 & $11.07 \pm 3.02$ & 1.94 \\
\hline
\end{tabular}

Table 2 Accuracy of estimates corresponding to three criteria 
Table 3 Estimates of mode shapes with uncertainty

\begin{tabular}{|c|c|c|c|c|c|c|}
\hline \multirow[t]{2}{*}{ Generator } & \multicolumn{2}{|c|}{ Local mode 1} & \multicolumn{2}{|c|}{ Local mode 2} & \multicolumn{2}{|l|}{ Inter-area } \\
\hline & Mag (p.u.) & Angle (rad) & Mag (p.u.) & Angle (rad) & Mag (p.u.) & Angle (rad) \\
\hline$\overline{G_{1}}$ & $1.00 \pm 0.06$ & $-0.27 \pm 0.03$ & $0.161 \pm 0.09$ & $2.60 \pm 0.18$ & $1.00 \pm 0.07$ & $2.88 \pm 0.11$ \\
\hline $\mathrm{G}_{2}$ & $0.982 \pm 0.06$ & $2.87 \pm 0.04$ & $0.154 \pm 0.07$ & $0.39 \pm 0.18$ & $0.694 \pm 0.10$ & $-2.84 \pm 0.10$ \\
\hline $\mathrm{G}_{3}$ & $0.159 \pm 0.07$ & $0.50 \pm 0.06$ & $1.00 \pm 0.08$ & $2.90 \pm 0.06$ & $0.794 \pm 0.07$ & $0.31 \pm 0.10$ \\
\hline $\mathrm{G}_{4}$ & $0.137 \pm 0.09$ & $2.76 \pm 0.12$ & $0.984 \pm 0.13$ & $-0.23 \pm 0.14$ & $0.85 \pm 0.08$ & $-0.26 \pm 0.19$ \\
\hline
\end{tabular}

where $\operatorname{sgn}(\cdot)$ is the sign function and $\widehat{u}_{s_{i j}}^{*}$ is the conjugate complex of $\widehat{u}_{s_{i j}}^{*}$. $G_{j}$ refers to the jth generator. The positive number $\mathcal{E}$ is the threshold. In this paper, $\mathcal{E}$ is set to be 0.3 . Thus, the conclusion can be drawn that the ith estimated mode is mainly caused by the generators in $M_{\mathrm{i}+}$ swinging against generators in $M_{\mathrm{i}_{-}}$. Comparing the conclusion with the results of modal analysis on firstprinciple modeling, one can determine which mode the estimated mode corresponds to.

Note that the mode matching method proposed in this paper can be simplified via aggregation model since an inter-area oscillation mode involves many generators. These generators can be divided into several coherent groups. Actually, coherence and aggregation, as a method of model reduction, is commonly used to analyze large power system dynamics. This kind of method is based on the relationship between the coherent areas and the slow inter-area modes [32]. It is not necessary to model the entire inter-connected power systems in detail to each generator when only several modes are of interest. One who has interest in the details may refer to [32] and [15].

\section{Simulation}

In this section, three test systems are used to illustrate the two improvements. Numerical algorithm for N4SID, one of the subspace identification methods, is applied in the simulation. In a steady state, a power system operates at an equilibrium point. The system is perturbed by load variations. These load variations can be regarded to follow Gaussian distribution. In [33], the loads of Nordic system are investigated statistically and it can be concluded that the loads are Gaussian distributed. In [34], power system disturbance behavior is also suggested to be modeled via Gaussian approach. In simulation tests, loads are modeled to follow the Gaussian distribution. Thus, the dynamic system with ambient noises is simulated. Measurement noises with 20-dB signal-to-noise ratio (SNR) are also taken into consideration.

\subsection{Four-Machine System}

The Four-Machine Test System shown in Figure 2 is used to demonstrate the accuracy of the improvements. There are 4 generators with governors and power system stabilizers (PSS) and 13 buses in this system. There are three low-frequency oscillation modes in the system. One is the inter-area mode with generators $G_{1}$ and $G_{2}$ swinging against generators $G_{3}$ and $G_{4}$. The other two are local mode 1 , the oscillation between generators $G_{1}$ and $G_{2}$ and local mode 2, the oscillation between generators $G_{3}$ and $G_{1}$.

Voltage angles of buses 1, 2, 10, and 20 are measured as system outputs. The sampling frequency is $0.2 \mathrm{~Hz}$ and data length $\mathrm{N}$ equals 3,000. Model order is first determined by AIC, BIC, and SVC, respectively. From Table 1, one can see that AIC and BIC determine the same system order so that the estimating results are the same while SVC suggests a small model order. One may find that the two local modes have very similar frequencies. Thus, the estimated modes cannot be decided to which modes of interest they correspond respectively. To this content, the proposed mode matching method can help tell the frequency neighboring modes apart. Mode shapes of each estimated mode are shown and compared with the reference ones in Figure 3. The match results are also shown in Table 1. From Figure 3

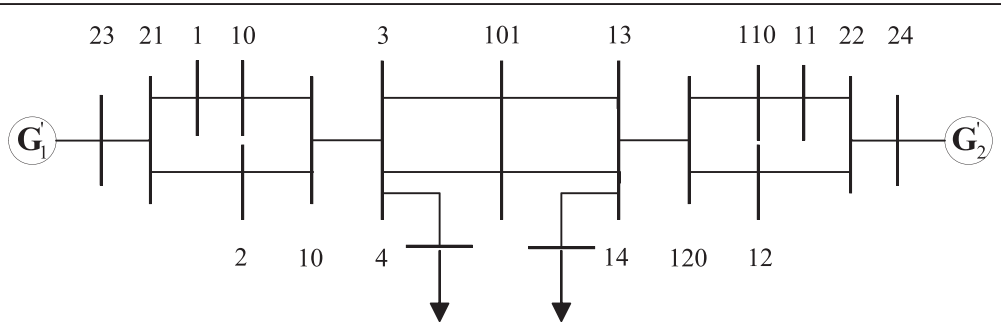

Figure 4 Schematic diagram of Four-Machine System after aggregation. 


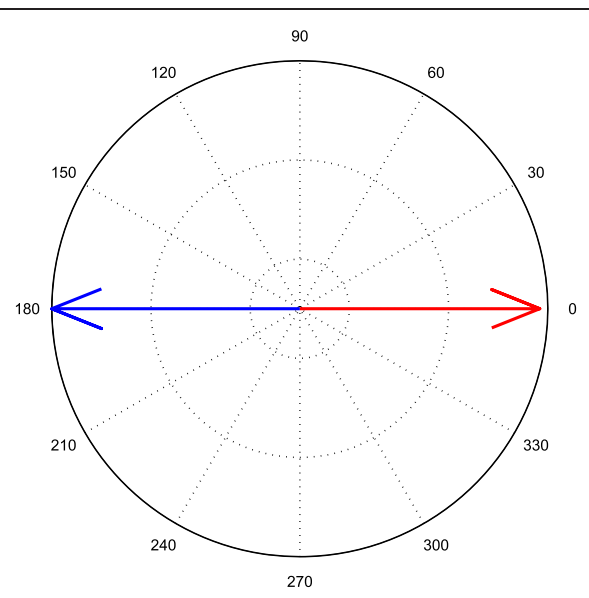

(a) reference

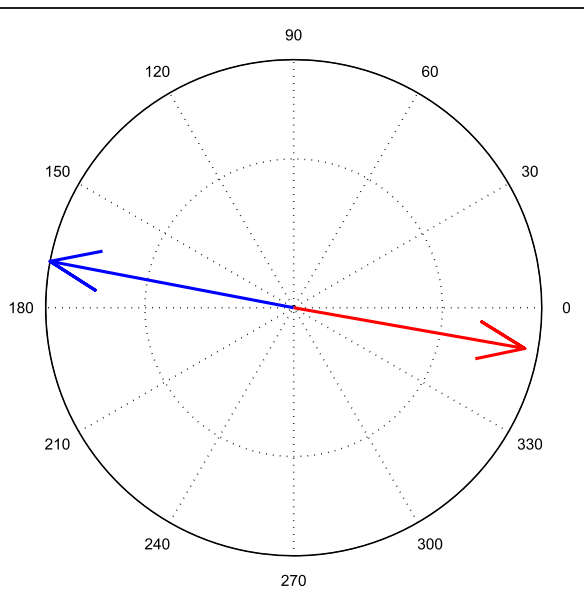

(b) estimated via aggregation model

Figure 5 Inter-are mode shapes of aggregation model. (a) Reference from aggregation model. (b) Estimated via aggregation model.

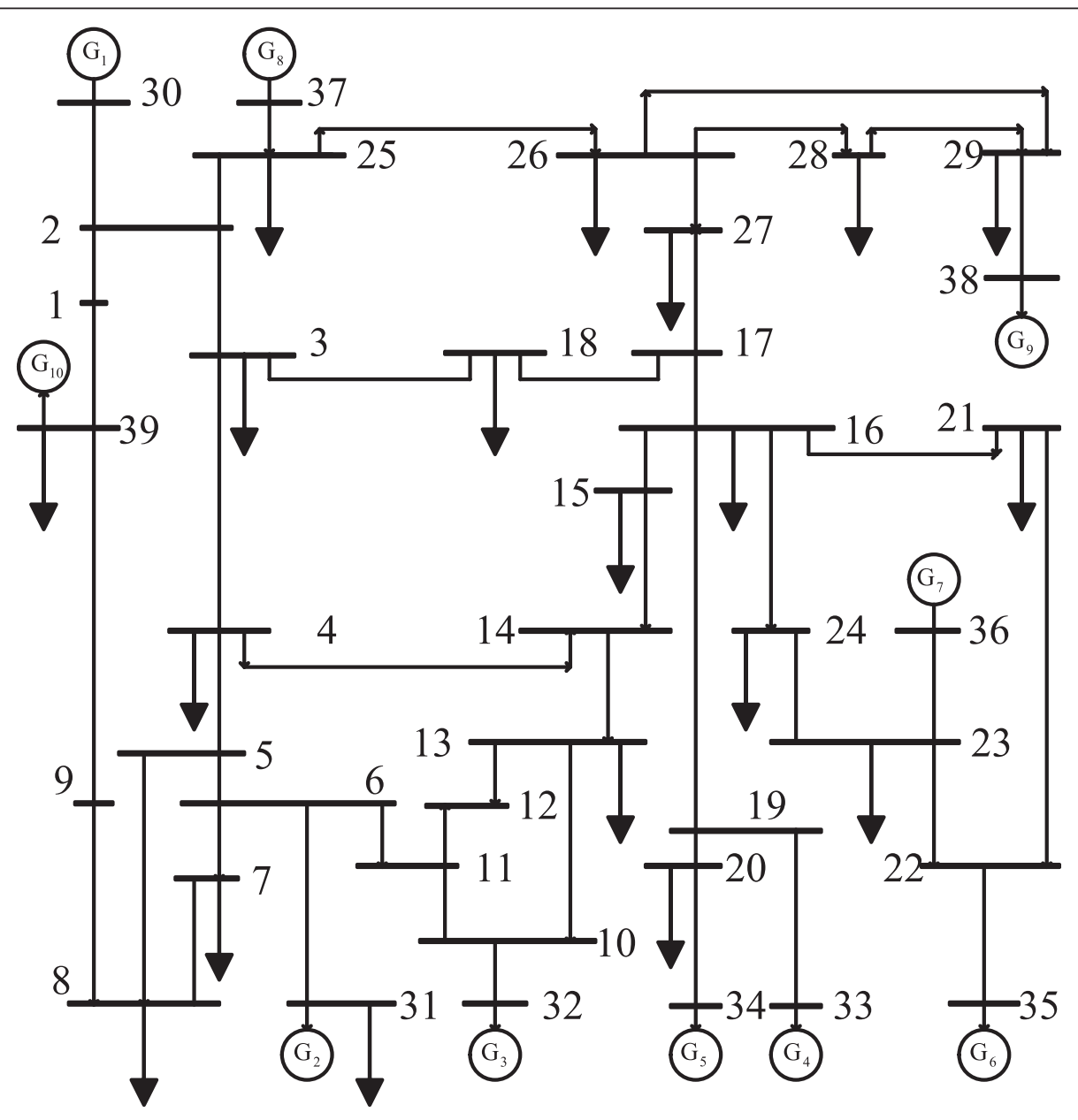

Figure 6 Schematic diagram of New England System. 
Table 4 Results of modal analysis for New England System

\begin{tabular}{|c|c|c|c|c|c|c|c|c|c|c|c|}
\hline \multirow[t]{2}{*}{ Mode } & \multirow[t]{2}{*}{ Freq $(\mathrm{Hz})$} & \multicolumn{10}{|c|}{ Eigenvectors of electromechanical oscillation modes } \\
\hline & & $\overline{\mathrm{G}_{1}}$ & $\mathrm{G}_{2}$ & $\mathrm{G}_{3}$ & $\mathrm{G}_{4}$ & $\mathrm{G}_{5}$ & $\mathrm{G}_{6}$ & $\mathrm{G}_{7}$ & $\mathrm{G}_{8}$ & $\mathrm{G}_{9}$ & $\mathrm{G}_{10}$ \\
\hline Local 1 & 1.021 & $+^{+}$ & - & - & - & - & - & - & + & + & + \\
\hline Local 2 & 1.139 & + & + & + & $+^{+}$ & $+^{+}$ & $-^{+}$ & $-^{+}$ & + & + & - \\
\hline Local 3 & 1.071 & + & $+^{\dagger}$ & $-^{\dagger}$ & + & + & + & + & + & - & - \\
\hline Local 4 & 1.170 & $-^{\dagger}$ & - & - & - & - & - & - & + & $+^{+}$ & - \\
\hline Local 5 & 1.134 & + & $+^{+}$ & $+^{\dagger}$ & - & - & - & - & + & + & - \\
\hline Local 6 & 1.311 & - & - & - & + & - & - & - & $+^{\dagger}$ & - & - \\
\hline Local 7 & 1.236 & + & - & - & + & - & - & $+^{+}$ & - & - & - \\
\hline Local 8 & 1.390 & + & - & - & $+^{+}$ & $-^{+}$ & + & - & - & - & + \\
\hline Inter-area & 0.636 & $+^{+}$ & $+^{\dagger}$ & $+^{\dagger}$ & $+^{+}$ & $+^{+}$ & $+^{+}$ & $+^{+}$ & $+^{\dagger}$ & $+^{+}$ & $-^{+}$ \\
\hline
\end{tabular}

and Table 1, it can be found that the modes can be correctly matched by proposed method.

Bootstrap method is applied to evaluate the accuracy of the estimates [8]. One thousand trials are generated for evaluation of model order selection. Table 2 shows the error bounds with $95 \%$ uncertainty of the estimates (mean \pm 2 standard deviation). Root mean square error (RMSE) is also calculated. From Table 2, it can be concluded that the uncertainties of the frequency and damping ratio estimates corresponding to AIC, BIC, and SVC are similar. But estimates corresponding to SVC have smaller RMSE which means SVC can give more accurate estimates.

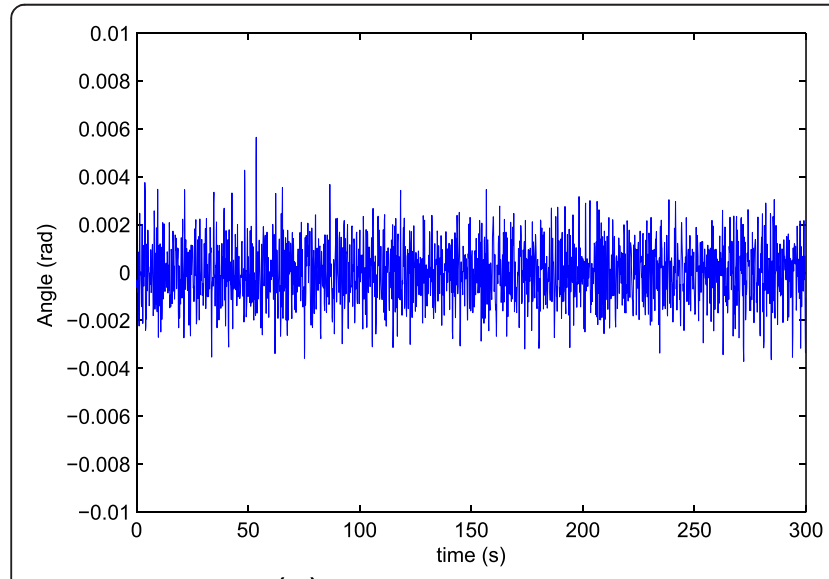

(a) voltage angle of Bus 1

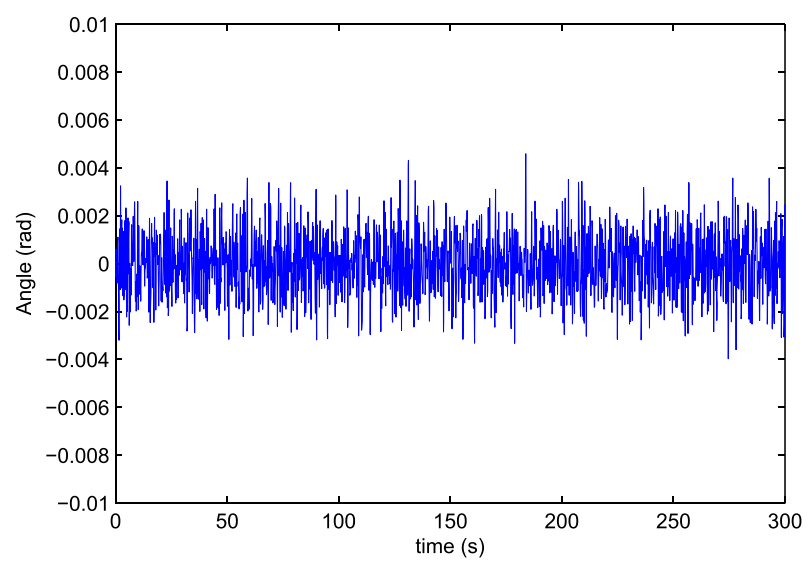

(c) voltage angle of Bus 5

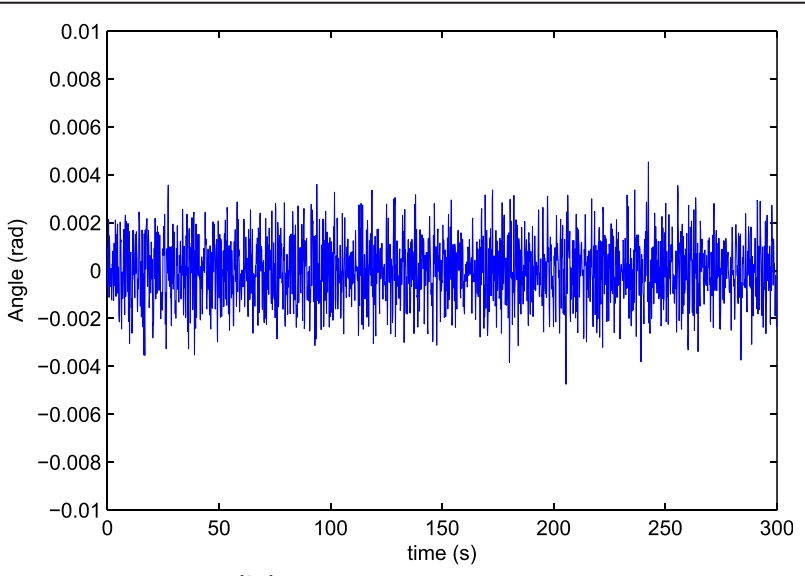

(b) voltage angle of Bus 3

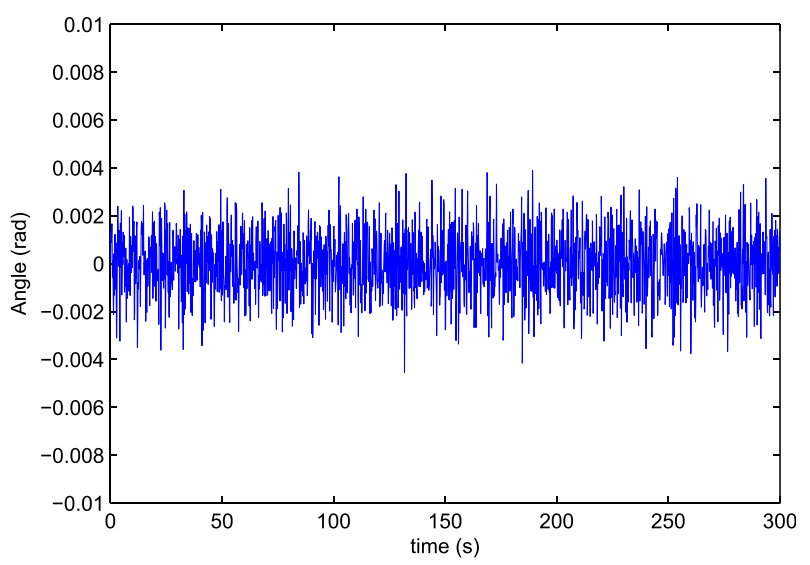

(d) voltage angle of Bus 15

Figure 7 Pre-processed signals. (a) Voltage angle of bus 1. (b) Voltage angle of bus 3. (c) Voltage angle of bus 5. (d) Voltage angle of bus 15. 
Also, the accuracy of mode matching is also evaluated. Table 3 shows the uncertainty of the estimated mode shapes in polar coordinates. One can see that the uncertainty of the estimates have no influence on the elements in $M_{\mathrm{i}+}$ and $M_{\mathrm{i}-\text {. }}$ Thus, the correctness of mode matching can be validated.

Practically, only several critical oscillation modes are of interest in some complicated systems. These modes are usually lightly damped inter-area mode in which many generators are involved. It would be very complicated to calculate the mode shapes on all the generators in the system. In this situation, the inertia aggregation method of coherent generators [32] can be applied to simplify the mode matching. The accuracy of mode matching for inter-area mode via aggregation method is also evaluated. The inertia aggregation method is applied to aggregate coherent generators $G_{1}$ and $G_{2}$ as generator $G_{1}$ and coherent generators $G_{3}$ and $G_{4}$ as generator $G_{2}$, respectively. The aggregated system is shown in Figure 4. The estimated and reference mode shapes of inter-area mode of aggregation system are shown in Figure 5.

From Figure 5a, one can obtain $M_{+}=\left\{\mathrm{G}_{2}^{\prime}\right\}$ and $M_{-}=$ $\left\{G_{1}^{\prime}\right\}$. This means that the estimated mode is caused by
$G_{1}$ swinging against $G_{2}$. Comparing the two figures, one can find that the estimated mode holds the same oscillation information as the inter-area mode, i.e., generators $\mathrm{G}_{1}$ and $G_{2}$ (generator $G_{1}$ ) swings against generators $G_{3}$ and $\mathrm{G}_{4}$ (generator $\mathrm{G}_{2}$ ). This means that the estimated mode is the inter-area mode. This sheds light on the fact that even by aggregation model, the mode matching method is still effective.

In Four-Machine System, the simulation results show that SVC can give more proper model order on which the accurate estimates of frequency and damping ratio are based. The estimated mode shapes are also accurate enough for mode matching. Furthermore, by aggregation methods, the proposed mode matching method can be both correct and simple.

\subsection{New England System}

For security analysis, there are only several modes of interest among many electromechanical oscillation modes in real power systems. The modes of interest will be interfered with some similar frequency modes out of interest. In order to show the flexibility of the improvements proposed in this paper, several modes of interest are shown to be estimated in the New England System

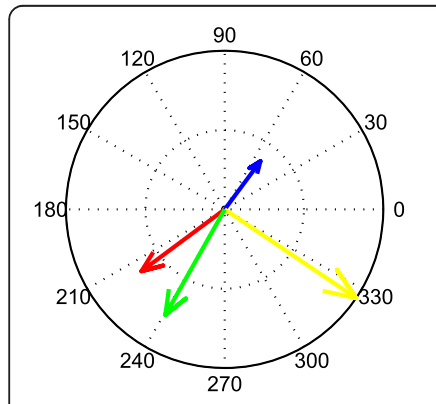

(a) estimated Mode 1

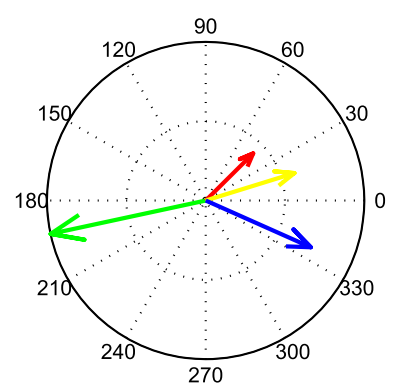

(e) estimated Mode 5

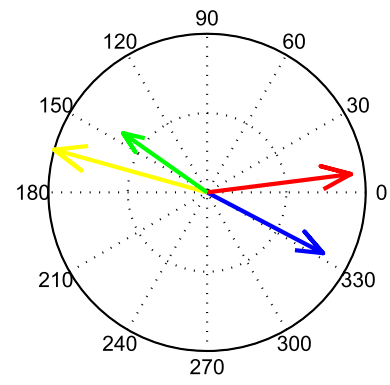

(b) Estimated Mode 2

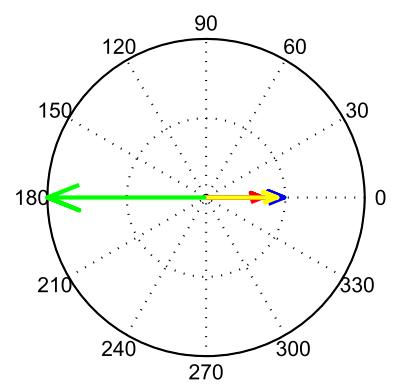

(f) Inter Area Mode

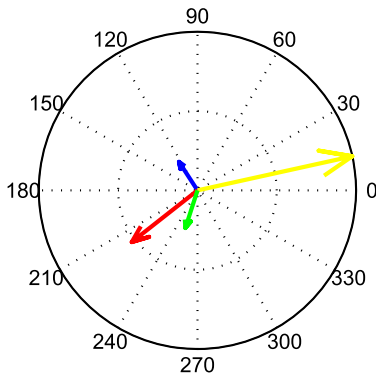

(c) estimated Mode 3

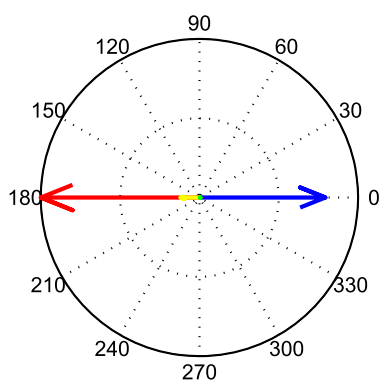

(g) Local Mode 1

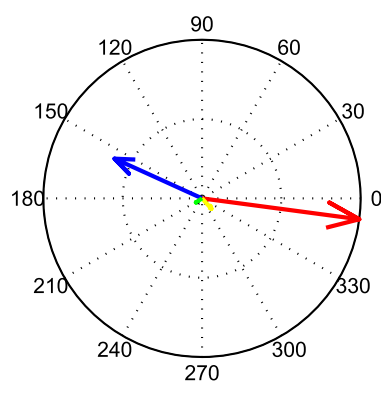

(d) estimated Mode 4

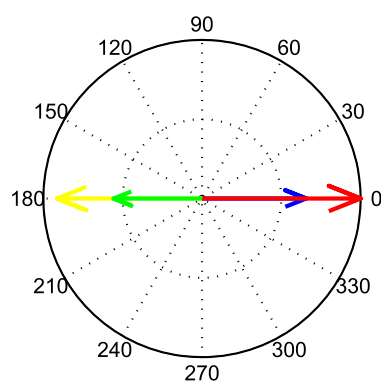

(h) Local Mode 5

\section{$-G^{\prime} 1-G^{\prime} 2=G^{\prime} 3=G^{\prime} 4$}

Figure 8 Reference and estimated mode shapes. (a) Estimated mode 1. (b) Estimated mode 2. (c) Estimated mode 3. (d) Estimated mode 4. (e) Estimated mode 5. (f) Inter-area mode. (g) Local mode 1. (h) Local mode 5. 
Table 5 Results of mode matching

\begin{tabular}{llll}
\hline Mode & \multicolumn{2}{l}{ Information from mode shapes } & Matching result \\
\cline { 2 - 3 } & $\boldsymbol{M}_{+}$ & $\boldsymbol{M}_{\mathbf{-}}$ & \\
\hline EST 1 & $G_{1}^{\prime} G_{3}^{\prime}$ & $G_{2}^{\prime} G_{4}^{\prime}$ & N/A \\
EST 2 & $G_{3}^{\prime} G_{4}^{\prime}$ & $G_{1}^{\prime} G_{2}^{\prime}$ & Local mode 5 \\
EST 3 & $G_{3}^{\prime}$ & $G_{1}^{\prime} G_{2}^{\prime} G_{4}^{\prime}$ & N/A \\
EST 4 & $G_{1}^{\prime} G_{4}^{\prime}$ & $G_{2}^{\prime} G_{3}^{\prime}$ & Local mode 1 \\
EST 5 & $G_{1}^{\prime} G_{2}^{\prime} G_{3}^{\prime}$ & $G_{4}^{\prime}$ & Inter-area mode \\
\hline
\end{tabular}

interfered with other frequency neighboring modes. The New England 39-bus system, shown in Figure 6, is composed of 39 buses and 10 generators. Generator $G_{10}$ is the equivalent external grid generator. The results of modal analysis are presented in Table 4. The ' + ' and '-' symbols indicate that the generators swing against each other. The symbol ' $t$ ' indicates that the generator mainly participates in the oscillation. For example, the first row indicates that local mode 1 is caused by generators $G_{1}$, $G_{8}, G_{9}$, and $G_{10}$ swinging against generators $G_{2}$ to $G_{7}$ and $G_{1}$ is mainly participated.

Local mode 1 , local mode 5 , and the inter-area mode are of interest. Note that local mode 1 and local mode 3 have similar frequencies and so are local mode 5 and local mode 4 (see Table 4). This means that the estimated frequencies can be of local mode 3 and local mode 4 or of local mode 1 and local mode 5 . How to correctly find out the modes corresponding to the ones of interest is shown. Take voltage angles of buses $1,3,5$, and 15 as system outputs at a data length of $\mathrm{N}(=1,500)$. Four pre-processed signals are illustrated in Figure 7.

Table 4 indicates that $G_{10}$ swings against the others in the inter-area mode, while $G_{1}, G_{9}$ and $G_{10}$ swing against the others in local mode $1 ; G_{1}, G_{2}, G_{3}, G_{8}$, and $G_{9}$ swing against $G_{4}, G_{5}, G_{6}, G_{7}$ and $G_{10}$ in local mode 5. Thus, one can aggregate generators in this way: $\left(G_{1}, G_{8}, G_{9}\right)$, $\left(G_{2}, G_{3}\right),\left(G_{4}, G_{5}, G_{6}, G_{7}\right)$ and $G_{10}$ can be aggregated to groups $G_{1}, G_{2}, G_{3}$, and $G_{4}$, respectively.

The model order is chosen to be 30 by SVC. Frequency, damping ratio, and corresponding mode shapes are estimated. There are five estimated modes whose frequencies are in between 0.4 and $1.5 \mathrm{~Hz}$. The proposed mode matching method is used to find out the modes corresponding to the ones of interest. Figure 8 illustrates the mode shapes of the five estimated modes as well as three reference ones. Mode matching results are shown in Table 5. From Table 5 and Figure 8, one can correctly match estimated mode 2 , estimated mode 4 , and estimated mode 5 with local mode 5 , local mode 1 , and inter-area mode, respectively. Thus, the estimate results can be determined in Table 5 .

Table 6 shows the estimates of local mode 1, local mode 5, and inter-area mode. The RMSE of the estimates are also calculated by bootstrap method and the results are listed in Table 6. From Tables 5 and 6, it can be concluded that the estimates are both correct and accurate.

\subsection{NPCC 48-machine system}

The proposed method can also be applied into a much larger power system, NPCC, containing many inter-area oscillation modes. The accuracy and flexibility will be shown in the system. The NPCC 48-machine system is composed of 140 buses and 48 machines in the system. The results of modal analysis show that there are eight inter-area modes and the whole system can be partitioned into nine areas. More details about the system description and coherent analysis can be found in [35]. The results of modal analysis are presented in Table 7 in which 'IM' stands for inter-area mode while ' $\mathrm{A}_{\mathrm{i}}$ ' stands for the generators in the $i$ th Area. The ' + ' and ' - ' symbols are also used to indicate that the generators swing against each other.

IM 6, IM 7, and IM 8 are the modes of interest due to their less damping ratios (see Table 7). Note that IM 7 and IM 8 have similar frequencies and one cannot tell the estimates apart only by the frequencies. Take voltage angles of buses $13,22,25,92,97,120,122,133$, and 139 as system outputs at a length of $\mathrm{N}(=3,000)$. The model order is chosen to be 26 by SVC. There are three estimated modes with frequencies between 0.65 and $0.90 \mathrm{~Hz}$. The proposed mode matching method is used to match the modes with the ones of interest, and the results of mode matching are shown in Table 8. From Table 8, one can find that the estimated modes 1, 2, and 3 are the estimated IM 6, IM 7, and IM 8, respectively. The estimate results can thus be determined in Table 8. It can be concluded that the estimates are correct and accurate.

\section{Conclusions}

Two improvements, model order selection and mode matching for estimating the frequencies, damping ratios and mode shapes of electromechanical oscillations via

Table 6 Estimates of local mode 1, local mode 5, and inter-area mode

\begin{tabular}{|c|c|c|c|c|c|c|}
\hline \multirow[t]{2}{*}{ Mode } & \multicolumn{3}{|c|}{ Frequency $(\mathrm{Hz})$} & \multicolumn{3}{|c|}{ Damping ratio (\%) } \\
\hline & Estimated & Reference & RMSE & Estimated & Reference & RMSE \\
\hline Local mode 1 & 1.042 & 1.021 & 0.023 & 4.74 & 5.31 & 1.70 \\
\hline Local mode 5 & 1.181 & 1.134 & 0.048 & 7.82 & 7.92 & 1.48 \\
\hline Inter-area mode & 0.631 & 0.636 & 0.009 & 3.82 & 3.98 & 1.45 \\
\hline
\end{tabular}


Table 7 Results of modal analysis for NPCC 48-machine system

\begin{tabular}{|c|c|c|c|c|c|c|c|c|c|c|c|}
\hline \multirow[t]{2}{*}{ Mode } & \multirow[t]{2}{*}{ Freq $(\mathrm{Hz})$} & \multirow[t]{2}{*}{ DR (\%) } & \multicolumn{9}{|c|}{ Eigenvectors of inter-area modes } \\
\hline & & & $A_{1}$ & $A_{2}$ & $A_{3}$ & $A_{4}$ & $A_{5}$ & $A_{6}$ & $A_{7}$ & $A_{8}$ & $\overline{A_{9}}$ \\
\hline$\overline{I M ~} 1$ & 0.256 & 16.67 & $+^{+}$ & $-^{\dagger}$ & + & + & - & - & + & $+^{\dagger}$ & + \\
\hline IM 2 & 0.381 & 11.69 & $-^{\dagger}$ & + & - & $+^{\dagger}$ & $-^{\dagger}$ & - & $+^{+}$ & + & $+^{+}$ \\
\hline $\operatorname{IM} 3$ & 0.472 & 8.39 & $-^{+}$ & - & + & - & + & - & + & + & - \\
\hline IM 4 & 0.530 & 7.90 & $-^{+}$ & + & - & $-^{\dagger}$ & - & + & $-^{+}$ & $+^{+}$ & $-^{+}$ \\
\hline IM 5 & 0.596 & 7.30 & $+^{+}$ & - & + & $-^{\dagger}$ & + & + & $-^{+}$ & - & $-^{+}$ \\
\hline IM 6 & 0.703 & 5.86 & + & + & + & $+^{\dagger}$ & - & - & $-^{+}$ & - & $+^{+}$ \\
\hline $1 \mathrm{M} 7$ & 0.704 & 6.45 & - & $+^{\dagger}$ & - & + & + & $-^{\dagger}$ & + & + & + \\
\hline IM 8 & 0.790 & 5.84 & - & + & + & $-^{\dagger}$ & + & - & - & - & $+^{+}$ \\
\hline
\end{tabular}

subspace identification methods have been presented in this paper. Based on theoretical analysis, both the two improvements are applied to simulation systems with ambient data. The results demonstrate that SVC works better than traditional information criteria in finding a proper system model order on which the accurate estimates of modal properties are based. The accuracy of the estimates has been investigated via bootstrap method. Also, with the help of aggregation model, the proposed mode matching method can easily help ensure the correctness of the estimates, even for larger power system. The emphasis of future work is placed upon investigating the influence of data length on the improved subspace identification methods and electromechanical mode tracking via subspace methods.

\section{Appendices}

\subsection{Appendix 1: Evaluation of entry of $\mathrm{K}_{\mathrm{S}}$}

The complex power $\tilde{S}_{i}$ for the ith generator in multimachine power system is as follows:

$$
\begin{gathered}
\tilde{S}_{i}=\dot{U}_{i} * \dot{I}^{*}{ }_{i} \\
=\underset{\substack{j=1 \\
j \neq i}}{N}\left|E^{\prime}{ }_{i}\right|\left|E^{\prime}{ }_{j}\right|\left|\mathbf{Y}_{\text {redij }}\right|\left(\cos \left(\delta_{i j}-\alpha_{i j}\right)+j \sin \left(\delta_{i j}-\alpha_{i j}\right)\right) \\
+\sum_{\substack{j=1 \\
j \neq i}}^{N}\left|E^{\prime}{ }_{i}\right|^{2}\left|\mathbf{Y}_{\text {redij }}\right|\left(\cos \alpha_{i j}-j \sin \alpha_{i j}\right)
\end{gathered}
$$

where $\mathrm{E}_{\mathrm{i}}^{\prime}$ is the voltage behind the transient reactance which is assumed to be constant. $\delta_{\mathrm{ij}}$ is the difference between $\delta_{i}$ and $\delta_{j} . \delta_{i}$ is the rotor angle of the $i$ th generator. $Y_{\text {redij }}$ is the admittance between the generators. $\alpha_{\mathrm{ij}}$ is the phase angle of the $\mathbf{Y}_{\text {redij}}$. $\mathbf{Y}_{\text {red }}$ can be calculated as follows:

$$
\mathbf{Y}_{\text {red }}=\mathbf{Y}_{m m}-\mathbf{Y}_{m_{n}} \mathbf{Y}_{n_{b} n_{b}}^{-1} \mathbf{Y}_{n_{b} m}
$$

Therefore, the electromagnetic power for the ith generator is as follows:

$$
\begin{aligned}
\mathrm{P}_{e i}=\operatorname{Re}\left\{\tilde{\mathrm{S}}_{i}\right\}= & -\sum_{\substack{j=1 \\
j \neq i}}^{N}\left|E^{\prime}{ }_{i}\right|\left|E^{\prime}{ }_{j}\right|\left|\mathbf{Y}_{\text {red } i j}\right| \cos \left(\delta_{i j}-\alpha_{i j}\right) \\
& +\sum_{\substack{j=1 \\
j \neq i}}^{N}\left|E^{\prime}{ }_{i}\right|^{2}\left|\mathbf{Y}_{\text {redij }}\right| \cos \alpha_{i j}
\end{aligned}
$$

\begin{tabular}{|c|c|c|c|c|c|c|c|}
\hline \multirow[t]{2}{*}{ Mode } & \multicolumn{2}{|c|}{ Information from mode shapes } & \multirow[t]{2}{*}{ Matching result } & \multicolumn{2}{|c|}{ Frequency $(\mathrm{Hz})$} & \multicolumn{2}{|c|}{ Damping ratio (\%) } \\
\hline & $\overline{M_{+}}$ & $\mathbf{M}_{-}$ & & Estimated & RMSE & Estimated & RMSE \\
\hline EST 1 & $\mathrm{~A}_{4} \mathrm{~A}_{9}$ & $A_{7}$ & IM 6 & 0.702 & 0.013 & 6.74 & 2.18 \\
\hline EST 2 & $A_{6}$ & $\mathrm{~A}_{2}$ & IM 7 & 0.705 & 0.012 & 7.51 & 2.28 \\
\hline EST 3 & $\mathrm{~A}_{4}$ & $A_{9}$ & IM 8 & 0.788 & 0.014 & 4.82 & 1.82 \\
\hline
\end{tabular}

After simplification

$$
\mathrm{P}_{e i}=-\sum_{\substack{j=1 \\ j \neq i}}^{N}\left|E^{\prime}{ }_{i}\right|\left|E^{\prime}{ }_{j}\right|\left|\mathbf{Y}_{\mathrm{red} i j}\right| \cos \left(\delta_{i j}-\alpha_{\mathrm{ij}}\right)+\left|E^{\prime}{ }_{i}\right|^{2} \mathrm{G}_{i i}
$$

Table 8 Results of mode matching 
Consequently, $\mathbf{K}_{\mathrm{s}_{\mathrm{ij}}}$, the value in the ith row and jth column of $\mathbf{K}_{s_{i j}}$, can be found as follows:

$$
\mathbf{K}_{\mathbf{s}_{i j}}=-\frac{\partial P_{e i}}{\partial \delta_{j}}=\left\{\begin{array}{l}
-\sum_{\substack{j=1 \\
j \neq i}}^{N}\left|E_{i}^{\prime}\right|\left|E_{j}^{\prime}\right|\left|\mathbf{Y}_{\text {redij }}\right| \sin \left(\delta_{i j}-\alpha_{i j}\right), i=j \\
\left|E_{i}^{\prime}\right|\left|E_{j}^{\prime}\right|\left|\mathbf{Y}_{\text {redij }}\right| \sin \left(\delta_{i j}-\alpha_{i j}\right), i \neq j
\end{array}\right.
$$

\subsection{Appendix 2: Computation of entry of $\mathbf{C}_{\theta \delta}$}

Referring to $\mathrm{m}$ generators as internal voltage nodes, one can get:

$$
\left[\begin{array}{c}
0 \\
\tilde{\mathbf{I}}_{\mathrm{m}}
\end{array}\right]=\left[\begin{array}{cc}
\tilde{\mathbf{Y}}_{\mathrm{n}_{\mathrm{b}} \mathrm{n}_{\mathrm{b}}} & \tilde{\mathbf{Y}}_{\mathrm{n}_{\mathrm{b}} \mathrm{m}} \\
\tilde{\mathbf{Y}}_{\mathrm{mn}_{\mathrm{b}}} & \tilde{\mathbf{Y}}_{\mathrm{mm}}
\end{array}\right]\left[\begin{array}{c}
\tilde{\mathbf{V}}_{\mathrm{n}_{b}} \\
\tilde{\mathbf{E}}_{\mathrm{m}}^{\prime}
\end{array}\right]
$$

where $\tilde{\mathbf{I}}_{\mathrm{m}}$ is the generator injection current matrix; $\tilde{\mathbf{E}}_{\mathrm{m}}^{\prime}$ is the generator internal voltage matrix; $\tilde{\mathbf{V}}_{\mathrm{n}_{\mathrm{b}}}$ is the node voltage matrix. Hence:

$$
\tilde{\mathbf{V}}_{\mathrm{n}_{\mathrm{b}}}=-\tilde{\mathbf{Y}}_{\mathrm{n}_{\mathrm{b}} \mathrm{n}_{\mathrm{b}}}{ }^{-} 1 \tilde{\mathbf{Y}}_{\mathrm{n}_{\mathrm{b}} \mathrm{m}} \tilde{\mathbf{E}}_{\mathrm{m}}^{\prime}
$$

Defining

$$
\tilde{\mathbf{K}} \triangleq-\tilde{\mathbf{Y}}_{\mathrm{n}_{\mathrm{b}} \mathrm{n}_{\mathrm{b}}}{ }^{-1} \tilde{\mathbf{Y}}_{\mathrm{n}_{\mathrm{b}} \mathrm{m}} \triangleq \mathbf{\kappa} \angle \mathrm{Y}
$$

results in

$$
\tilde{V}_{i}=\sum_{j=1}^{\mathrm{m}} \tilde{\boldsymbol{\kappa}}_{i j} \tilde{E}_{j}^{\prime}=\sum_{j=1}^{\mathrm{m}} \boldsymbol{\kappa}_{i j}\left|\tilde{E}_{j}^{\prime}\right| \angle\left(\gamma_{i j}+\delta_{j}\right)
$$

Expanding (37)

$$
\tilde{V}_{i}=\sum_{\mathrm{j}=1}^{\mathrm{m}} \boldsymbol{\kappa}_{\mathrm{ij}}\left|\tilde{E}_{j}^{\prime}\right| \cos \left(\delta_{j}+\gamma_{i j}\right)+j \sum_{j=1}^{\mathrm{m}} \boldsymbol{\kappa}_{i j}\left|\tilde{\mathrm{E}}_{j}^{\prime}\right| \sin \left(\delta_{j}+\gamma_{i j}\right)
$$

results in

$$
\theta_{i}=\arctan \frac{\sum_{j=1}^{\mathrm{m}} \boldsymbol{\kappa}_{i j}\left|\tilde{E}_{j}^{\prime}\right| \sin \left(\delta_{j}+\mathrm{Y}_{i j}\right)}{\sum_{j=1}^{\mathrm{m}} \boldsymbol{\kappa}_{i j}\left|\tilde{E}_{j}^{\prime}\right| \cos \left(\delta_{j}+\mathrm{Y}_{i j}\right)}
$$

Defining

$$
\begin{aligned}
\left|\tilde{V}_{i}\right| \triangleq & \left(\left(\sum_{j=1}^{\mathrm{m}} \boldsymbol{\kappa}_{i j}\left|\tilde{E}_{j}^{\prime}\right| \cos \left(\delta_{j}+\mathrm{Y}_{i j}\right)\right)^{2}\right. \\
& \left.+\left(\sum_{j=1}^{\mathrm{m}} \boldsymbol{\kappa}_{i j}\left|\tilde{E}_{j}^{\prime}\right| \sin \left(\delta_{j}+\mathrm{Y}_{i j}\right)\right)^{2}\right)^{\frac{1}{2}}
\end{aligned}
$$

Results in

$$
\begin{aligned}
& \frac{\partial \theta_{\mathrm{i}}}{\partial \delta_{\mathrm{j}}}=\frac{1}{1+\left(\frac{\sum_{q=1}^{\mathrm{m}} \boldsymbol{\kappa}_{i q}\left|\tilde{E}_{q}^{\prime}\right| \sin \left(\delta_{q}+\mathrm{Y}_{i q}\right)}{\sum_{j=1}^{\mathrm{m}} \boldsymbol{\kappa}_{i q}\left|\tilde{E}_{q}^{\prime}\right| \cos \left(\delta_{q}+\mathrm{Y}_{i q}\right)}\right)^{2}} * \\
& \left(\frac{\left(\sum_{q=1}^{\mathrm{m}} \boldsymbol{\kappa}_{i q}\left|\tilde{E}_{q}^{\prime}\right| \sin \left(\delta_{q}+\mathrm{Y}_{i q}\right)\right) \kappa_{i j}\left|\tilde{E}_{j}^{\prime}\right| \sin \left(\delta_{j}+\mathrm{Y}_{i j}\right)}{\left(\sum_{q=1}^{\mathrm{m}} \boldsymbol{\kappa}_{i q}\left|\tilde{E}_{q}\right| \cos \left(\delta_{q}+\mathrm{Y}_{i q}\right)\right)^{2}}\right. \\
& \left.+\frac{\boldsymbol{\kappa}_{i j}\left|\tilde{E}_{j}^{\prime}\right| \cos \left(\delta_{j}+\mathrm{Y}_{i j}\right)}{\sum_{q=1}^{\mathrm{m}} \boldsymbol{\kappa}_{i q}\left|\tilde{E}_{q}^{\prime}\right| \cos \left(\delta_{q}+\mathrm{Y}_{i q}\right)}\right)
\end{aligned}
$$

i.e.,

$$
\begin{aligned}
\frac{\partial \theta_{i}}{\partial \delta_{j}}= & \frac{1}{\left|\tilde{V}_{i}\right|^{2}}\left(\sum_{q=1}^{\mathrm{m}} \boldsymbol{\kappa}_{i q}\left|\tilde{E}_{q}^{\prime}\right| \sin \left(\delta_{q}+\gamma_{i q}\right) \boldsymbol{\kappa}_{i j}\left|\tilde{E}_{j}^{\prime}\right| \sin \left(\delta_{j}+\gamma_{i j}\right)\right. \\
& \left.+\sum_{q=1}^{\mathrm{m}} \boldsymbol{\kappa}_{i q}\left|\tilde{E}_{q}^{\prime}\right| \cos \left(\delta_{q}+\gamma_{i q}\right) \boldsymbol{\kappa}_{i j}\left|\tilde{E}_{j}^{\prime}\right| \cos \left(\delta_{j}+\gamma_{i j}\right)\right)
\end{aligned}
$$

Finally, one can conclude the following:

$$
\frac{\partial \theta_{i}}{\partial \delta_{j}}=\frac{1}{\left|\tilde{V}_{i}\right|^{2}}\left(\sum_{q=1}^{\mathrm{m}} \boldsymbol{\kappa}_{i q} \boldsymbol{\kappa}_{i j}\left|\tilde{E}_{q}^{\prime}\right|\left|\tilde{E}_{j}^{\prime}\right| \cos \left(\delta_{q}+\mathrm{Y}_{i q}-\delta_{j}-\mathrm{Y}_{i j}\right)\right)
$$

\section{Competing interests}

Both authors declare that they have no competing interests.

\section{Acknowledgment}

This research was supported in part by Prof Y M Shi of Zhejiang University.

\section{Author details}

${ }^{1}$ The state key laboratory of industrial control technology, Institute of Cyber-Systems and Control, College of Control Science and Engingeering, Zhejiang University, Hangzhou 310027, China. ${ }^{2}$ College of Electrical Engineering, Zhejiang University, Hangzhou 310027, China. 


\section{References}

1. X Zhou, J Yi, R Song, X Yang, Y Li, H Tang, An overview of power transmission systems in China. Energy 35(11), 4302-4312 (2010). doi:10.1016/j.energy.2009.04.016

2. Z Liang, M Xiao, K Zhang, J Zhou, J Wu, Discussion on control strategy for low frequency oscillation in China Southern Power Grid. Automation of Electric Power Syst 35(11), 54-58 (2011)

3. Canadian Electrical Association Report, Investigation of low frequency inter-area oscillation problems in large inter-connected power systems, Ontario Hydro, 1993

4. P Kundur, Power system security in the new industry environment: challenges and solutions. (IEEEE Toronto Section, 2003), http://www.ewh.ieee.org/r7/ toronto/events/oct0303/prabha.ppt

5. Final Report on the August 14, 2003 Blackout in the United States and Canada: causes and recommendations (U.S.-Canada Power System Outage Task Force, Tech. Rep., 2004)

6. SJ Qin, An overview of subspace identification. Comput Chem Eng 30(10-12), 1502-1513 (2006). doi:10.1016/j.compchemeng.2006.05.045

7. N Zhou, JW Pierre, RW Wies, Estimation of low-frequency electromechanical modes of power systems from ambient measurements using a subspace method, in Proceedings of the 35th North American Power Symposium, Rolla, 2003

8. N Zhou, Dissertation (University of Wyoming, Laramie, 2005)

9. DJ Trudnowski, JW Pierre, N Zhou, JF Hauer, M Parashar, Performance of three mode-meter block-processing algorithms for automated dynamic stability assessment. IEEE Trans Power Syst 23(2), 680-690 (2008). doi:10.1109/TPWRS.2008.919415

10. L Dosiek, N Zhou, JW Pierrre, Z Huang, DJ Trudnowski, Mode shape estimation algorithms under ambient conditions: A comparative review. IEEE Trans Power Syst 28(2), 779-787 (2013). doi:10.1109/ TPWRS.2012.2210570

11. R Shibata, Selection of the order of an autoregressive model by Akaike's information criterion. Biometrika 63(1), 117-126 (1976). doi:10.1093/ biomet/63.1.117

12. G Schwarz, Estimating the dimension of a model. Ann Statist 6(2), 461-464 (1978). doi:10.1214/aos/1176344136

13. C Wu, C Lu, Y Han, New algorithm for mode shape estimation based on ambient signals considering model order selection. EURASIP Journal on Advances in Signal Processing. 2013(8), (2013). doi: 10.1186/1687-6180-2013-8

14. D Bauer, Dissertation (TU Wien, Vienna, 1998)

15. L Wang, M Klein, S Yirga, P Kundur, Dynamic reduction of large power systems for stability studies. IEEE Trans on Power Syst 12(2), 889-895 (1997). doi:10.1109/59.589749

16. IEEE Task Force Report, Identification of electromechanical modes in power systems (The Institute of Electrical and Electronics Engineers, Inc, 2012

17. G Liu, VM Venkatasubramanian, JR Carroll, Oscillation monitoring system using synchrophasors, in IEEE Proceeding of PES General Meeting, Calgary, 2009, pp. 1-4

18. L Dosiek, Dissertation (University of Wyoming, Laramie, 2010)

19. JY Cai, Z Huang, JW Hauer, K Martin, Current status and experience of WAMS implementation in North America, in IEEE Proceeding of Transmission and Distribution Conference and Exhibition: Asia and Pacific, Dalian, 2005, pp. 1-7

20. AG Place, GH Allen, Generalized pole sensitivity analysis due to parameter perturbation. IEEE Trans Circuits Syst II, Analog Digit Signal Process 44(10), 869-873 (1997). doi:10.1109/82.633448

21. L Vanfretti, Dissertation (Rensselaer Polytechnic Institute, Troy, 2009)

22. TJ Turunen, J Thambirajah, M Larsson, BC Pal, NF Thornhill, LC Haarla, WW Hung, AM Carter, T Rauhala, Comparison of three electromechanical oscillation damping estimation methods. IEEE Transactions on Power Syst 26(4), 2398-2407 (2011). doi:10.1109/TPWRS.2011.2155684

23. S Bittanti, M Lovera, Bootstrap-based estimates of uncertainty in subspace identification methods. Automatica 36(11), 1605-1615 (2000). doi: 10.1016/ S0005-1098(00)00081-9

24. MG Anderson, N Zhou, JW Pierre, RW Wies, Bootstrap-based confidence interval estimates for electromechanical modes from multiple output analysis of measured ambient data. IEEE Trans Power Syst 20(2), 943-950 (2005). doi:10.1109/TPWRS.2005.846125

25. L Dosiek, JW Pierre, An improved bootstrap method for electromechanical mode estimation using multivariate probability distributions, in IEEE Proceeding of PES General Meeting, San Diego, 2011, pp. 1-8
26. N Zhou, JW Pierre, D Trudnowski, A bootstrap method for statistical power system mode estimation and probing signal selection, in IEEE Proceeding of PES General Meeting, Montreal, 2006, pp. 1-7

27. H Akaike, Information theory and an extension of the maximum likelihood principle, in Proceeding of 2nd International Symposium on Information Theory, Tsahkadsor, 1971, pp.267-281

28. D Bauer, Order estimation for subspace methods. Automatica 37(10), 1561-1573 (2001). doi: 10.1016/S0005-1098(01)00118-2

29. L Ljung, System Identification: Theory for the User, 2nd edn. (Prentice-Hall, Inc., Englewood Cliffs, NJ, 1987)

30. P Kundur, Power System Stability and Control (McGraw Hill, New York, 1994)

31. P Kundur, GJ Rogers, DY Wong, L Wang, MG Lauby, A comprehensive computer program package for small signal stability analysis of power systems. IEEE Trans Power Syst 5(4), 1076-1093 (1990). doi:10.1109/59.99355

32. JH Chow, Power System Coherency and Model Reduction (Springer, New York, 2013)

33. J Turunen, M. Larsson, J. Thambirajah, LC. Haarla, T. Rauhala, Recent Developments in Modal Estimation of Power System Electromechanical Oscillations, in IEEE Proceeding of PowerTech, Trondheim, 2011, pp. 1-7

34. DN Ewart, Power: whys and wherefores of power system blackouts: an examination of the factors that increase the likelihood and the frequency of system failure. IEEE Spectr 15(4), 36-41 (1978). doi:10.1109/MSPEC.1978.6367667

35. J Chow, PV Kokotovic, RJ Thomas (eds.), Systems and Control Theory for Power Systems (Springer, New York, 1995)

\section{Submit your manuscript to a SpringerOpen ${ }^{\odot}$ journal and benefit from:}

- Convenient online submission

- Rigorous peer review

- Immediate publication on acceptance

- Open access: articles freely available online

- High visibility within the field

- Retaining the copyright to your article

Submit your next manuscript at springeropen.com 